\title{
Improving hospital weekend handover: a user-centered, standardised approach
}

\author{
Avi Mehra, Christin Henein \\ Brighton \& Sussex University Hospitals NHS Trust
}

\begin{abstract}
Clinical Handover remains one of the most perilous procedures in medicine (1). Weekend handover has emerged as a key area of concern with high variability in handover processes across hospitals $(1,2,4,5-10)$.

Studying weekend handover processes within medicine at an acute teaching hospital revealed huge variability in documented content and structure. A total of 12 different pro formas were in use by the medical day-team to handover to the weekend team on-call. A Likert-survey of doctors revealed $93 \%$ felt the current handover system needed improvement with $71 \%$ stating that it did not ensure patient safety (Chisquared, $p$-value $<0.001, n=32$ ). Semi-structured interviews of doctors identified common themes including "a lack of consistency in approach" "poor standardization" and "high variability".
\end{abstract}

Seeking to address concerns of standardization, a standardized handover pro forma was developed using Royal College of Physician (RCP) guidelines (2), with direct end-user input. Results following implementation revealed a considerable improvement in documented ceiling of care, urgency of task and team member assignment with $100 \%$ uptake of the new proforma at both 4-week and 6-month post-implementation analyses. $88 \%$ of doctors surveyed perceived that the new proforma improved patient safety $(p<0.01, n=25)$, with $62 \%$ highlighting that it allowed doctors to work more efficiently. Results also revealed that $44 \%$ felt further improvements were needed and highlighted electronic solutions and handover training as main priorities.

Handover briefing was subsequently incorporated into junior doctor induction and education modules delivered, with good feedback. Following collaboration with key stakeholders and with end-user input, integrated electronic handover software was designed and funding secured. The software is currently under final development.

Introducing a standardized handover proforma can be an effective initial step in improving weekend handover. Handover education and enduser involvement are key in improving the process. Electronic handover solutions have been shown to significantly increase the quality of handover and are worth considering $(9,10)$.

\section{Problem}

Clinical Handover in hospitals remains one of the most perilous procedures in medicine (1). A process that is embedded in the healthcare system and essential for the delivery of safe patient care, the challenge of enabling safe handover is experienced on an international, national and institutional level. Handover has been identified as a point at which errors in communication, prioritisation and subsequent actions are likely to occur and failure of handover is a major preventable cause of patient harm (2).

The introduction of the European Working Time Directive (EWTD) in the UK has led to an increased shift pattern of work for junior doctors, with subsequently more points at which patient information and responsibility of care must be transferred or handed over (3). This is especially important during preparation for the weekend oncall where junior doctors are often tasked with anticipating and handing over patient information and jobs for the whole weekend. The concept of removing the 'weekend shift' and ensuring the same standard of healthcare is available $24 / 7$ is a worthy one, however until such progress is made one must increase awareness of the weekend handover as a critical point at which errors can occur and ensure improvements are implemented to prevent subsequent patient harm. Weekend handover practices vary considerably between hospitals with a combination of paper based and electronic modalities in use. The introduction and use of a standardized handover pro forma has been shown to improve the efficiency of general data transfer during patient handover (4).

Studying weekend handover practices in the department of medicine at an acute teaching hospital in England revealed huge variability in patient information transferred between the week and weekend on-call. Different medical teams and wards were found to be using multiple different handover pro formas to communicate information to the weekend on-call team. Furthermore, no training was delivered to the junior doctors on how to complete the handover pro forma effectively and what information was necessary to include. Personal experience of a delay in the review of a patient who required regular fluid balance assessment, caused partly by omission of information in the weekend handover pro forma, including failure to assign a team-member, document urgency of the task and document adequate task information, highlighted a 
clear issue with weekend handover processes and prompted further action to improve the system. Multiple doctors of different grades had come forward to express their desire to improve the current weekend handover system with growing frustration at the lack of any meaningful or sustainable change. Recognizing the inadequacies in the current system, a team of doctors formed the Handover Improvement Initiative; a trust-wide grassroots team with the aim of improving clinical handover.

\section{Background}

Improving weekend handover is an area tackled regularly throughout various hospitals and trusts $(5,6)$. Studies have highlighted significant adverse events resulting from inadequate handover $(5,7)$. Handover education and training, standardized proformas and electronic software have all emerged as key areas to improve weekend handover (1,2,5-10). The National Patient Safety Agency has highlighted poor quality handover as a significant risk to patient safety (1) and the General Medical Council's (GMC) Good medical practice has expressed a clear need for doctors to keep their colleagues well informed about patient care, especially in current working patterns (11). Recent data published by Dr Foster Intelligence identified a worrying $10 \%$ spike in death rates over the weekend compared to weekdays (12), with sub-optimal handover highlighted as a major preventable cause of patient harm (2). However despite much media attention, development of guidelines (1) and efforts to improve the handover process, many hospitals are still failing to effectively address this key issue. In the most recent GMC annual survey (2013) $20 \%$ of 54,000 junior doctors stated that handover arrangements before and after night duty were informal or that no arrangements were made (13). It is clear that more needs to be done to improve clinical handover at weekends but also during the week. A collaborative, system-wide approach harnessing cultural, technological and educational fixes is essential to implementing sustainable transformative change to ultimately improve internal handover processes and thereby improve patient safety $(1,2)$.

\section{Baseline Measurement}

Working general medical weekend on-calls it was clear that medical teams were using different handover pro formas to transfer important patient information and responsibility of care to the weekend team. The weekend handover pro formas were stored and archived securely on a shared drive, which were therefore accessible for review and audit. The baseline was divided into three steps.

Firstly, an audit of the weekend handover pro forma was carried out using data from one random weekend on-call. The audit was carried out in the general medicine department by three junior doctor colleagues (FY1-FY2). The general medicine department had been highlighted as the major area of concern regarding weekend handover processes and was subsequently a first priority. The audit revealed the following findings. $A$ total of 12 different pro formas were in use by the medical day-team to hand over patients to the medical weekend team on-call. The 12 pro formas in use all had different layouts with varied patient information and handover content. 9 out of the 12 were in a table format whilst 3 were simply in a list format with patient information, medical issues and allocated tasks all in different formats and structures. The pro formas were then saved on the shared drive separate to the patient's notes and nursing records. Only medical doctors working in general medicine had access to the shared drive. Upon arrival on the Saturday morning the weekend team were then expected to print out all the different pro formas and navigate through them to decipher what jobs needed to be done and in what order.

During the weekend in review a total of 82 patients were handed over to the weekend team on-call. $87 \%$ of patients had a minimum of three patient identifiers documented (minimum of 3 patient identifiers required in guidance by Royal College of Physicians (2), $12 \%$ had two and $1 \%$ had one. Only $1 \%$ of patients had a documented ceiling of care within the handover content with $0 \%$ stating the urgency or priority of the weekend task. Only $63 \%$ had a clearly documented team member who the weekend task was allocated to (e.g. F1, SHO or Registrar). There was a distinct lack of consistency between pro formas with the only shared items present in all versions being patient identifiers (name, date of birth and hospital number) and tasks to be done. All other items including documented team member, urgency of task, patient ceiling of care and patient location varied considerably in their presence amongst all pro formas.

Secondly, recognizing this variability and following concerns expressed by fellow colleagues regarding the current weekend handover practice, a likert-type survey was developed and disseminated amongst non-consultant doctor colleagues (FY1-Reg). These grades were identified as the major users in the current handover process and were subsequently chosen for sampling. The focus of the survey was to further explore the views and concerns regarding handover practices, held by direct users within the handover process (namely FY1-Reg doctors), and to suggest possible solutions.

The Likert-type survey included a total of nine questions with likertscales and free text answers. The survey was developed and disseminated amongst 32 medical doctors (ranging from FY1 Registrar).

Finally, In conjunction with the survey, 30 semi-structured interviews of Registrar doctors were carried out by a Leadership Registrar through a collaborative leadership project called 'The Network' to further decipher common themes.

Following completion, the surveys were collected and the data analysed. $93 \%$ of the doctors surveyed felt the current weekend handover process needed improvement and $71 \%$ thought that it did not ensure patient safety (Chi-squared, $p$-value $<0.001, n=32$ ). $81 \%$ thought the current system did not allow doctors to work efficiently and $90 \%$ also highlighted that there was not adequate handover training or teaching at induction.

Data from the semi-structured interviews were analysed using Access-coding software and Word clouds were generated. $51 \%$ of 
comments regarding Handover were not positive and the major themes identified were "a lack of consistency in approach" "poor standardization" and "high variability".

See supplementary file: ds2643.docx - "Likert-type Survey"

\section{Design}

The baseline measurements helped identify areas of concern in the current weekend handover process and acted as a catalyst for the formation of The Handover Improvement Initiative (HII). HII was formed initially by junior doctors, however following recruitment it comprised of a team covering a range of doctors representing all grades (FY1- Consultant) who had been directly involved in weekend handover. FY1, SHO and Registrar doctors were responsible for completing and using the weekend handover pro forma, attending all shift handover meetings and coordinating the majority of weekend handover arrangements. Consultant doctors had oversight of the system as a whole and were also involved in specific handover activities. The Chief of Patient Safety was recruited to supervise and further develop the initiative.

Following analysis of the baseline measurements, and in response to requests for standardization and improvements to the handover process, a standardized handover pro forma was developed using guidelines outlined by the Royal College of Physicians (RCP) (2).

Audit of weekend handover pro formas revealed that medical teams and wards were using different handover pro formas with subsequent high variability in the content of transferred information. This meant the on-call team working the weekend had to print off different handover lists for each ward/ medical team in different formats containing different information. This lack of standardization was naturally confusing for the on-call team and provided very little structure with how to tackle the requested tasks.

The development of a new standardized pro forma, to be used by all medical teams and wards across medicine, aimed to provide consistency in approach and improve efficiency for the on-call team over the weekend. The aim was to avoid increasing the workload for either the day-team or on-call team and as such criteria outlining the development of the new pro forma highlighted that it be easy to use, contained key appropriate information and ensured a standardized approach across the department.

The new pro forma was developed to include key sets of information including a minimum of 3 patient identifiers, location, medical issues, ceiling of care, Priority (Urgent / Non-urgent), task assignment including on-call member who task is assigned to and day of requested task. This was developed in line with RCP guidelines and with constant collaboration and agreement between team members. The proforma was to be stored in a single handover folder on a shared drive so that all information was easily accessible. A pre-filled pro forma was developed to act as a guideline, which was also stored in the same shared folder.

From the baseline results it was clear immediate change was needed and so following development the pro forma was launched across the whole medical department for immediate use on a pre- assigned weekend. In order to achieve complete uptake it was essential that a structured approach was adopted in the launch. Firstly, a short information session was delivered at the mandatory junior doctor teaching session one week prior to pro forma release. Using a power-point presentation, doctors were guided through the new pro forma layout, provided with information on how to complete the pro forma effectively and had any questions or queries answered. The doctors were informed of the pre-assigned release date and the location of the pro forma in the shared drive. To supplement this information session and to account for doctors who were not present an e-mail outlining the new changes was disseminated across the trust. One week after the teaching session, the new weekend handover pro forma was implemented. This was done on a Friday and team members were assigned to visit each ward personally to ensure all medical teams were utilizing the new pro forma and to provide assistance if required.

Four weeks post implementation of the new pro forma a re-audit was carried out. This involved a further Likert-type survey of junior doctors to assess response to the new intervention and analysis of the archived proformas in the shared drive.

A further audit of the archived proformas was carried out 6 months post implementation to further assess proforma usage and sustainability of improvements. Through the Handover Improvement Initiative specific team-members were given responsibility for auditing usage and effectiveness of the new pro forma at 4-week and 6-month post-implementation points, with further plans in place to audit every 4 months from there on. Team-members responsible for this area were also tasked with handing over this responsibility upon leaving the hospital to a new team-member, to ensure sustainability of improvements and facilitate continuous ongoing measurements.

\section{Strategy}

During the first improvement cycle, the standardised weekend handover pro forma was shown to doctor colleagues (end-users of the pro forma) and was rigorously analysed. This included ensuring the pro forma was in line with guidelines outlined by the Royal College of Physicians, contained the appropriate information and was simple to use. Minor changes were made to the original version, including re-defining 'urgency of task' to either Urgent or Non-urgent and eliminating the use of 'routine' which was highlighted as an unfavourable choice. During the second cycle the standardised pro forma was implemented across the general medicine department. The usage and effectiveness of the pro forma was assessed 4-weeks and 6-months post-implementation. With evidence of sustained $100 \%$ uptake and with significant improvements in documented content at both assessment points, no changes to the pro forma layout or content were highlighted as necessary and the pro forma layout remained unchanged. However, during the second cycle, in response to additional data gathered, other areas were targeted including improving handover education and developing integrated electronic handover software.

\section{Results}


The weekend pro formas were re-audited four weeks post implementation with the following results. During the weekend studied all medical teams were using the one standardized proforma with full $100 \%$ uptake. No other pro formas were in use. A total of 76 patients were handed over to the weekend team on-call. $95 \%$ of patients had a minimum of three patient identifiers documented, $4 \%$ had two and $1 \%$ had one. $45 \%$ of patients had a clearly documented ceiling of care with $67 \%$ stating the urgency of the weekend task. $97 \%$ had a clearly documented team member who the weekend task was allocated to. The results showed a clear improvement compared to baseline analysis.

A second Likert-type survey of 25 doctors was also carried out four weeks post-implementation. Results showed that $88 \%$ of doctors surveyed perceived that the new pro forma improved patient safety (Chi-squared $p<0.01, n=25$ ), with $62 \%$ highlighting that the new pro forma allowed doctors to work more efficiently.

Results from the survey also revealed that $44 \%$ of doctors felt weekend handover needed further improvement with the majority highlighting the need for permanent electronic solutions. Further training and handover education were also identified as popular requests. In response to these findings the Handover Improvement Initiative met to discuss strategies to improve the process further. Additional individuals including chiefs of acute medicine and education were recruited to discuss realistic sustainable solutions to improve weekend handover across the trust. Following lengthy discussions and analysis of the issues at hand, an agreement was reached to research the possibility of developing an integrated electronic handover software to ultimately replace the paper-based pro formas.

In order to assess the sustainability and ongoing usage of the standardized pro forma whilst further improvements were made, a further audit of the weekend prof ormas was carried out 6 months post implementation. Pro formas from a weekend on-call were accessed and studied. Results revealed that again all medical teams were still using the one standardized pro forma with no other pro formas in use. There was no variation or adaptation of the standardized pro forma with all teams using the exact same template. During the weekend in review, a total of 88 patients were handed over to the weekend team on call. $98 \%$ had a minimum of three patient identifiers documented and $2 \%$ had minimum of two. $60 \%$ of patients had a clearly documented ceiling of care with $88 \%$ stating the urgency of the weekend task. $100 \%$ of patients handed over had a clearly documented team member who the weekend task was allocated to. These results showed a clear improvement in compliance with RCP guidelines compared to 1-month postimplementation analysis. They also highlighted a sustained uptake of the standardised pro forma evidenced by $100 \%$ ongoing uptake. Through sustained improvements in weekend handover documentation content, standardisation of the process, increased awareness of weekend handover processes and regular system analysis, patient safety was subsequently improved $(1,2)$.

\section{Lessons and Limitations}

Improving weekend handover is a complex endeavour. Despite increasing evidence of improvements made nationwide, the process still presents complex challenges that need to be addressed to ensure sustainable effective solutions are implemented.

Introducing a standardized handover pro forma in line with RCP guidelines can be an effective initial step in improving weekend handover. It provides a cheap, easy to implement solution that can be easily adapted and modified according to local needs.

The project also highlighted the importance of utilising a structured approach when attempting to launch a new initiative. Through formation of a project group representative of all grades of doctors involved in handover, a collaborative approach was adopted to tackle the issue. It was essential to ensure open communication with end users to facilitate maximum involvement and uptake. Gathering support for such an initiative also posed its own hurdles. Senior involvement from an early stage proved pivotal in breaking down barriers within existing hierarchical structures and networks, whilst utilisation of trust activities and forums, such as 'The Innovation Forum', helped increase awareness of the issues and gather colleague support.

Assessment of improvements to patient safety and doctor efficiency was based primarily on subjective assessment of the doctors involved in handover and is therefore one limitation of the study. Pro formas could also have been analysed over multiple weekends to increase the audited sample size.

We recognized the paper-based proforma had its own limitations and would realistically only act as an intermediate step. It still posed issues regarding patient confidentiality, task accountability and paperwork load. Listening to the local needs and through collaboration with key stakeholders the team attempted to overcome these challenges through developing integrated handover software. Although the pro forma only focused on the medical department it was clear challenges in clinical handover were faced in all departments, both during the weekend and during the week. Hence, the software was developed with full functionality across all specialties with usability on weekday, weekend and bank holiday shifts.

Development of permanent integrated handover software was initiated following collaboration with key stakeholders, including IT, Finance, senior management and software developers. Electronic handover software has been shown to significantly improve the quality of handover, providing better continuity of care, increased number of completed fields and flexibility in distribution of tasks compared with paper-based handover $(9,10)$. Development commenced and following a successful business case application, funding was secured from an education fund. The Agile Software Development process was used to direct development of the handover software and Functional Design. This was developed with direct end-user input, using an iterative incremental process spanning over 7 months. Important features include continuity of key information, prioritisation of tasks and accountability of jobs in real-time. It also allows for filtering by patients suitable for discharge so that patients are easily identifiable by the responsible registrar to 
ultimately improve weekend discharge processes. Filter functions for Medicine, surgery and specialties enable it to be utilised trustwide. The software is currently under final development and is planned for launch in 2014.

In response to the request for further training and education, handover briefing was incorporated into junior doctor induction and education modules were delivered. The modules were initially given to final year medical students with good feedback and plans were included to expand to include further years and junior doctors. Education and handover training are key to the development of safe and effective handover practices (1). Numerous requests were received for handover training and subsequently additional procedures were put in place to deliver this effectively.

Following further discussions within the Handover Improvement Initiative, a separate project was initiated to improve the 'hand-back' of patients following night shifts through the development of a Baton Bleep system. Discussions were also had regarding whether improvements could be made to the handover meeting process and doctors' shift pattern structure. Other aspects of the handover process not addressed by this study including bedside nursing handovers, inter-site handovers and patient perspectives require further research and development locally. These areas are all under assessment and part of future development plans.

\section{Conclusion}

This study showed a considerable improvement in documentation of task urgency, ceiling of care, team member assignment and documentation of a minimum of three patient identifiers following the implementation of a standardised weekend handover pro forma. Utilising a collaborative approach throughout the project ensured sustainability of improvements, with continued $100 \%$ uptake of the new pro-forma. User analysis also highlighted a significant improvement in perception of patient safety and doctor efficiency following implementation. Formation of The Handover Improvement Initiative helped increase awareness of the issues at hand, connect with key stakeholders, and facilitate development of further improvements to the handover process.

Introducing a standardized handover proforma can be an effective initial step in improving weekend handover. It can help to ensure all doctors, regardless of ward or sub-team, work with a common structured approach thereby reducing variability. It is essential to involve the end-users to ensure any new intervention is tailored to local needs. In order to implement sustainable effective change a system wide approach combining technological, cultural and system fixes is essential and this requires more research and development. Handover education and training is also key in addressing this critical area. Electronic handover solutions have been shown to significantly improve the quality of handover and are worth considering to improve processes further $(9,10)$.

\section{References}

Guidance on clinical handovers for patients and managers. London, http://bma.org.uk/-/media/Files/PDFs/Practical\%20advice\%2 0at\%20work/Contracts/safe\%20handover\%20safe\%20patie nts.pdf

2. Royal College of Physicians. Acute Care Toolkit 1: Handover (May 2011).

http://www.rcplondon.ac.uk/sites/default/files/acute-caretoolkit-1-handover.pdf

3. The Royal College of Surgeons of England (2007) Safe handover: guidance from the Working Time Directive working party (RCS, London) www.rcseng.ac.uk/publications/docs/publication.2007-05-14 .3777986999/.

4. Ferran NA, Metcalfe AJ, O'Doherty D. Standardised proformas improve patient handover: Audit of trauma handover practice. Patient safety surg. 2008; 2: 24

5. Pfeffer PE, Nazareth D, Main N, Hardoon S, Choudhury $\mathrm{AB}$. Are weekend handovers of adequate quality for the oncall general medical team? Clinical Medicine 2011, Vol 11, No 6: 536-40

6. Curtis $\mathrm{O}$, Fisher R. Improving medical $\mathrm{SHO}$ weekend handover at a tertiary referral centre. BMJ Quality Improvement Reports 2013;2

7. Horwitz LI, Moin T, Krumholz HM, Wang L, Bradley EH. Consequences of inadequate sign-out for patient care. Arch Internal Medicine 2008; 168: 1755-60

8. Govier M, Medcalf P. Living for the weekend: electronic documentation improves patient handover. Clinical Medicine 2012, Vol 12, No 2: 124-7

9. Raptis DA, Fernandes C, Chua W, Boulos PB. Electronic software significantly improves quality of handover in a London teaching hospital. Health Inform J 2009;15(3):191e8.

10. Ryan S, O'Riordan, JM, et al. Impact of a new electronic handover system in surgery. International Journal of Surgery 2011; 9(3): 217-220

11. General Medical Council. Good medical practice. London. (2013)

12. Dr Foster Intelligence: Hospital Guide (2011) http://drfosterintelligence.co.uk

13. General Medical Council (GMC) Annual Survey 2013. http://www.gmc-uk.org/news/23045.asp

\section{Declaration of interests}

None declared

\section{Acknowledgements}

In particular thanks to Dr Philippa Guppy, Mr Mark Edwards, Dr Prashant Parulekar, Dr Stephen Drage and Bhavna Sapat for their significant contribution to this Initiative. 